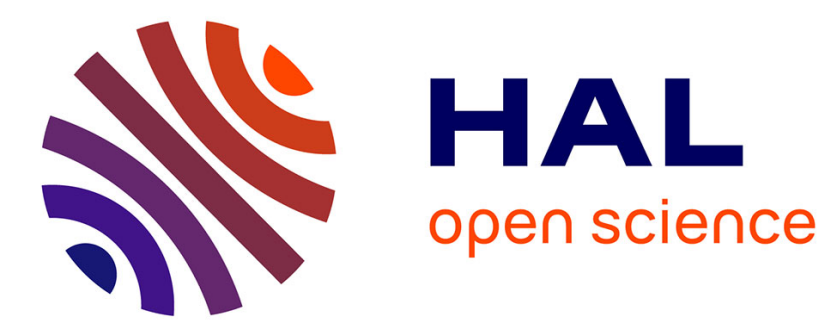

\title{
Impact of EIRP Constraint on MU-MIMO 802.11ac Capacity Gain in Home Networks
}

Khouloud Issiali, Valéry Guillet, Ghaïs El Zein, Gheorghe I. Zaharia

\section{To cite this version:}

Khouloud Issiali, Valéry Guillet, Ghaïs El Zein, Gheorghe I. Zaharia. Impact of EIRP Constraint on MU-MIMO 802.11ac Capacity Gain in Home Networks. Mediterranean Conference on Information \& Communication Technologies (MEDICT) 2015, May 2015, Saïdia, Morocco. pp.75-84, 10.1007/9783-319-30301-7_9. hal-01149441

\section{HAL Id: hal-01149441 https://hal.science/hal-01149441}

Submitted on 7 May 2015

HAL is a multi-disciplinary open access archive for the deposit and dissemination of scientific research documents, whether they are published or not. The documents may come from teaching and research institutions in France or abroad, or from public or private research centers.
L'archive ouverte pluridisciplinaire HAL, est destinée au dépôt et à la diffusion de documents scientifiques de niveau recherche, publiés ou non, émanant des établissements d'enseignement et de recherche français ou étrangers, des laboratoires publics ou privés. 


\title{
Impact of EIRP Constraint on MU-MIMO 802.11ac Capacity Gain in Home Networks
}

\author{
Khouloud Issiali ${ }^{1}$, Valéry Guillet ${ }^{1}$, Ghais El Zein ${ }^{2}$, Gheorghe Zaharia ${ }^{2}$, \\ Orange Labs, 1 Rue Louis et Maurice de Broglie, 90007 Belfort Cedex, France \\ ${ }^{2}$ IETR - INSA, UMR 6164, 20 av. des buttes de Coësmes, CS 70839, \\ 35708 Rennes Cedex 7, France \\ \{ Khouloud Issiali, Valery Guillet\}@orange.com \\ \{Ghais.El-Zein, Gheorghe.Zaharia\}@insa-rennes.fr
}

\begin{abstract}
In this paper, we evaluate a downlink Multi-User Multiple-Input Multiple-Output (MU-MIMO) scenario, in which a 802.11ac access point with multiple antennas (up to 10) is transmitting to two receivers, each one with two antennas. Block diagonalization (BD) method is investigated under the Equivalent Isotropic Radiated Power (EIRP) constraint. This study shows that scaling the transmitted power according to the EIRP constraint can improve the multi-user (MU) sum capacity to single-user (SU) capacity ratio compared to the gain achieved under the transmitted power constraint.
\end{abstract}

\section{Introduction}

Techniques such as MU-MIMO in IEEE802.11ac systems are proposed to increase the throughput up to $1 \mathrm{Gbps}$ [1]. These techniques consist of applying a linear precoding to the transmitted spatial streams. Consequently, the antenna array pattern and gain are modified as functions of the user location and propagation channel properties. This directly impacts the Equivalent Isotropic Radiated Power (EIRP). The European regulation sets the EIRP limit in the $5 \mathrm{GHz}$ frequency bands to 200 $\mathrm{mW}$ or $1 \mathrm{~W}$ depending on the propagation channels. This limit differs in other countries where it is defined based on the total transmitted power.

The EIRP constraint is rarely evaluated in the literature for MIMO systems. In most of the MIMO performance results, the packet error rate or the capacity value is evaluated based on the same total transmitted power $\left(P_{T x}\right)$ which is a function of the Signal to Noise Ratio $(S N R)$. The used $S N R$ is commonly defined as the ratio of the average total transmitted power to the average noise power. The propagation channel is usually normalized to have an average path loss of $0 \mathrm{~dB}$. Few recent studies have focused on the capacity optimization problems under total transmitted (Tx) power constraint [2-4]. This optimization is sometimes performed on each subcarrier of the 802.11 OFDM signal [2]. In [5], a new EIRP-based solution for IEEE 802.11 power scaling is proposed. However, this study is dedicated to only one single user system with a single spatial stream. 
The linear precoding applied for MU-MIMO processing, like Block Diagonalization (BD) [3], [6], modifies dynamically the antenna array pattern and gain. This changes the EIRP of the Tx antenna array if Tx power remains unchanged. MU-MIMO and Transmit Beamforming (TxBF) are commonly associated with a large number of Tx antennas used to improve the antenna array gain and performance, as previously stated for narrowband i.i.d Rayleigh SISO channels forming the MIMO channel in [4], [6]. In the case of the EIRP constraint, it may not be evident that TxBF and MU-MIMO linear precoding still improve the system performance.

Therefore, this paper evaluates the impact of the EIRP constraint on 802.11ac MUMIMO capacity gain using Matlab simulations. Two different power allocation schemes of the spatial streams are analyzed to optimize MU-MIMO capacity: equal and unequal power repartition under the same EIRP constraint. A typical indoor residential environment is evaluated based on the IEEE TGac correlated channels [7], [8]. Comparisons are given versus an i.i.d. Rayleigh coefficients.

The rest of this paper is organized as follows. Section 2 describes the system model and briefly presents the BD algorithm. The problem formulation to compute the sum capacity for MU-MIMO system under the EIRP constraint is given in Section 3. Section 4 describes the simulation process and presents the simulation results with analysis. Finally, the conclusion is drawn in Section 5.

We briefly summarize the notation used throughout this article. Superscripts $(.)^{t},(.)^{*}$ and $\left(^{-}\right)$denote the transposition, transpose conjugate and the complex conjugate, respectively. Expectation (ensemble averaging) is denoted by $\mathrm{E}($.$) .$ The Frobenius norm of a matrix is written as||. ||. Index $k$ is used as a user index and it runs from 1 to $K$, where $K$ is the number of users in the studied system.

\section{System Model}

\subsection{System Overview}

The studied 802.11ac MU-MIMO system is composed of $K$ users connected to one Access Point (AP) as shown in Fig. 1. The access point has $n_{T}$ antennas and each user $k$ has $n_{R_{k}}$ receiving antennas. We define $n_{R}=\sum_{k=1}^{K} n_{R_{k}}$. The $L_{k} \times 1$ (where $L_{k}$ is the number of parallel symbols transmitted simultaneously for the $k^{\text {th }}$ user) transmit symbol vector $s$ is preprocessed at the access point before being transmitted. For each 802.11ac OFDM subcarrier, the received signal at the $k^{\text {th }}$ receiver is given by:

$$
y_{k}=H_{k} W_{k} s_{k}+H_{k} \sum_{i=1, i \neq k}^{K} W_{i} s_{i}+n_{k}
$$

where $H_{k}$ is an $n_{R_{k}} \times n_{T}$ matrix that refers to the MIMO channel matrix for the $k^{\text {th }}$ receiver, $n_{k}$ is the noise vector composed of complex Gaussian noise $\left(E\left(n_{k} n_{k}^{*}\right)=\right.$ $\left.\sigma_{n}^{2} I_{n_{R_{k}}}\right)$, and $W_{k}$ is the BD precoding matrix $\left(n_{T} \times L_{k}\right)$ intended to the $k^{\text {th }}$ user resulting in a precoding matrix $W=\left[W_{1}, \ldots, W_{K}\right]$ The size of $W$ is $\mathrm{n}_{\mathrm{T}} \mathrm{x} \mathrm{L}$, with $\mathrm{L}=\mathrm{L}_{1}+\mathrm{L}_{2}+\ldots+\mathrm{L}_{\mathrm{K}}$ 


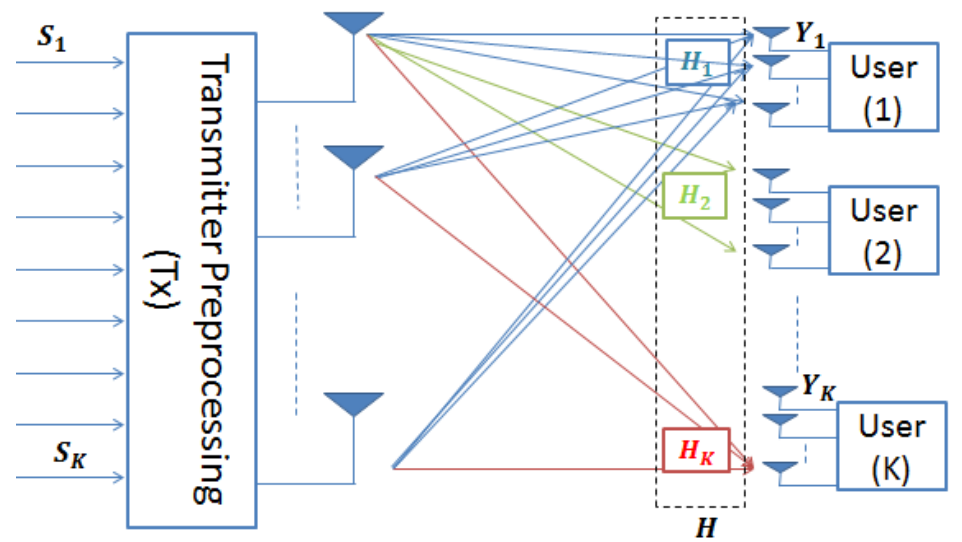

Fig. 1. Diagram of MU-MIMO system.

Block diagonalization [4], [6] is a transmit preprocessing technique for downlink MU-MIMO systems. BD decomposes the MU-MIMO downlink system into $K$ parallel independent SU-MIMO downlink systems. The BD method consists first of perfectly suppressing the inter-user interference (IUI) (IUI $=H_{k} \sum_{i=1, i \neq k}^{K} W_{i} s_{i}$ ) in order to have parallel SU-MIMO systems. Then, a classic TxBF is applied to optimize the capacity for each user [9]. In this study, we set $K=2$, but the results and algorithms can be generalized to any $\mathrm{K}$ number of users. Furthermore, we assume perfect knowledge of channel state information at the transmitter.

\subsection{Channel Model}

MU-MIMO performance is related to the propagation channel properties. In this study, the channel model specified for the 802.11ac standard within the TGac task group [8] is selected. This channel model takes into account realistic transmit and receive correlation contrary to an i.i.d Rayleigh channel. It is based on a cluster model [7] amended by the TGac task group for the IEEE 802.11ac standard. The TGac modifications concern the power angular spectrum to allow MU-MIMO operation and are summarized as follows [8]:

- The defined TGn azimuth spread for each cluster remains the same for all users.

- For each user, independent random offsets between $+/-180^{\circ}$ are introduced for the angle of arrival (AoA), the direct tap and the Non Line Of Sight NLOS taps.

- For each user, independent random offsets between $+/-30^{\circ}$ are introduced for the angle of departure (AoD), the direct tap and the NLOS taps.

In this study, a typical home network is evaluated by using the channel model TGac-B (15 ns RMS delay spread) for the $5.25 \mathrm{GHz}$ frequency band. Rayleigh fading is exhibited for each one of the 9 uncorrelated taps, except for the Line Of Sight (LOS) 
tap which follows a Rice fading with a $0 \mathrm{~dB}$ Rician factor. This study focuses on the TGac-B NLOS channel model. Similar results are obtained with the TGac-B LOS channel model as the $0 \mathrm{~dB}$ Rician factor does not display significantly different results from the TGac-B NLOS channel model. For each 802.11ac OFDM subcarrier, the channel matrix is computed through a discrete Fourrier transformation (size: 56 subcarriers) of the tap delay representation. For comparison, an i.i.d Rayleigh channel is also evaluated.

We apply the common normalization $E\left(\|H\|^{2}\right)=n_{T} n_{R}$ for each subcarrier which means an average propagation loss equal to $0 \mathrm{~dB}$.

\section{Problem Statement}

\subsection{Usual Definitions}

The MU-MIMO system is decomposed into $\mathrm{K}$ independent SU-MIMO systems by applying the BD algorithm. For each one of the 802.11ac OFDM subcarriers, the MUMIMO sum capacity is expressed as follows [4]:

$$
C_{B D}=\sum_{k=1}^{K} \sum_{i=1}^{n_{R_{k}}} \log _{2}\left(1+\frac{p_{i k}}{\sigma_{n}^{2}} \mu_{i k}^{2}\right)
$$

where $p_{i k}$ is the power dedicated to the $i^{\text {th }}$ antenna for the $k^{\text {th }}$ user, $\mu_{i k}^{2}$ are the eigenvalues of the effective channel for the $k^{\text {th }}$ user after applying the IUI cancellation [3] and $\sigma_{n}^{2}$ is the noise power. The subcarrier index is not mentioned throughout this paper in order to simplify the notations, but since $C_{B D}$ is related to $H, C_{B D}$ depends on each OFDM subcarrier.

For the corresponding SU-MIMO systems and for relevant comparisons with MUMIMO, the number of antennas $n_{T}$ and $n_{R}$ remains unchanged. The considered SUMIMO system applies a singular value decomposition and its capacity $C_{S U}$ is computed as detailed in [9] for each OFDM subcarrier.

\subsection{EIRP in Linear Precoding}

For any receiver location, i.e. for any $H$ matrix, the transmit antenna array pattern is modified by the $\mathrm{W}$ precoding matrix. We have used a linear array of omnidirectional 0 $\mathrm{dBi}$ gain antennas with a regular spacing $\delta$, typically $\delta=\lambda / 2$. The transmitter antenna array, manifold $a(\theta)$ as a function of the $\theta$ angle with the array axis, is defined as:

$$
a(\theta)^{t}=\left(1, e^{-2 j \pi \delta \cos (\theta) / \lambda}, e^{-4 j \pi \delta \cos (\theta) / \lambda}, \ldots, e^{-2 j \pi\left(n_{T}-1\right) \delta \cos (\theta) / \lambda}\right) .
$$

In practice with real antennas, a 3D antenna pattern can be included in this processing. Since the used TGac channel model is only a 2D model, the antenna array pattern expression is simplified to a $2 \mathrm{D}$ problem. The average radiated power $d(\theta)$ in 
any direction $\theta$ relative to the antenna array direction is expressed as a function of the input signals $x^{t}=\left(x_{1}, \ldots, x_{n_{T}}\right)$ :

$$
d(\theta)=E\left(\left|a(\theta)^{t} x\right|^{2}\right) .
$$

With $x=W s$, the radiated power contribution of any subcarrier is simply expressed as a function of the transmit diagonal correlation matrix $B=E\left(\bar{S} S^{t}\right)=\operatorname{diag}\left(p_{i k} ; k=1, \ldots, K ; i=1, \ldots, n_{R_{k}}\right)$ as:

$$
d(\theta)=a(\theta)^{*} \bar{W} B W^{t} a(\theta) .
$$

Considering all the subcarriers of the 802.11 ac system, the total radiated power $d(\theta)$ is expressed as:

$$
d_{\text {total }}(\theta)=\sum_{\text {subcarrier }} d(\theta)
$$

and the EIRP is:

$$
E I R P=\max _{\theta} d_{\text {total }}(\theta)
$$

In the particular case in which the total power $P_{T x}$ is equally shared among the spatial streams, we have $p_{i k}=\frac{P_{T x}}{N S S}$ where NSS denotes the total number of spatial streams. Thus, $d(\theta)$ can be simplified as:

$$
d(\theta)=\frac{P_{e}}{N S S} a(\theta)^{*} \bar{W} B W^{t} a(\theta) .
$$

\subsection{Optimization Problems}

The capacity optimization process consists of finding the optimal value of the Tx power $p_{i k}$ compatible with the EIRP constraint. Considering the spatial streams, two power allocation schemes are evaluated: equal power allocation and unequal power allocation. For practical reasons, such as the computation time, this paper focused on the case where each subcarrier has the same allocated total Tx power. Furthermore, an unequal subcarrier power allocation may not have a favorable impact on the peak-toaverage power ratio of the OFDM signal. The general optimization problem is thus expressed for each subcarrier as:

$$
\left\{\begin{array}{c}
\max _{p_{i k}} \sum_{k=1}^{K} \sum_{i=1}^{n_{R_{k}}} \log _{2}\left(1+\frac{p_{i k}}{\sigma_{n}^{2}} \mu_{i k}^{2}\right) \\
\text { and } E I R P \leq 23 \mathrm{dBm} .
\end{array}\right.
$$

To find the optimal solution of such a convex problem, the optimization is performed using a Matlab-based modelling for convex optimization namely CVX 
[10]. A simpler optimization problem consists of solving the case with equally distributed powers, i.e. $p_{i k}=\frac{P_{T x}}{N S S}$. This optimization problem has only one variable $P_{e}$. The problem is simplified by seeking the maximum antenna array gain $\frac{d(\theta)}{P_{T x}}$ and then scaling the power according to the EIRP limit. The case $K=1$ uses the same optimization method for computing the SU-MIMO capacity for both equal and unequal power allocation under EIRP constraint.

\subsection{Evaluated Systems and SNR Considerations}

802.11ac MU-MIMO systems based on BD schemes are evaluated. The results are presented in Section 4 and are compared to SU-MIMO systems relying on the same antennas and total power or EIRP constraint. Three capacity optimization techniques are evaluated and compared. The first one is the usual MIMO system (denoted basic), with a constant Tx power $P_{T x}$ equally shared among the spatial streams. BD-basic and $S U$-basic denote the corresponding studied systems. For this case, the average signal to noise ratio $S N R$ is defined as $S N R=\frac{P_{T x}}{\sigma_{n}^{2}}$. This is the common $S N R$ definition adopted in most of the published MIMO capacity studies.

The second optimization considers a $23 \mathrm{dBm}$ EIRP constraint and a total Tx power equally shared among the spatial streams. This optimization is labelled eirp-equal. A dynamic power scaling is applied, as detailed above, as a function of each channel matrix snapshot $H$. SUeirp-equal and BDeirp-equal denote respectively the corresponding $\mathrm{SU}$ and $\mathrm{MU}$ systems applying this technique.

The third one (eirp-unequal) considers a $23 \mathrm{dBm}$ EIRP constraint and a total Tx power unequally and dynamically shared among the spatial streams. SUeirp-unequal and BDeirp-unequal denote the corresponding systems applying this technique.

For eirp-equal and eirp-unequal systems, the common $S N R$ definition is biased as $P_{T X}$ is no more constant, and depends on each channel matrix computation. We subsequently define the average $S N R_{\text {eirp }}$ under $E I R P$ constraint as $S N R_{\text {eirp }}=\frac{E I R P}{\sigma_{n}^{2}}$ for eirp-equal and eirp-unequal systems.

Note that the maximum antenna array gain is $n_{T}$. Since $S N R=\frac{P_{T x}}{\sigma_{n}^{2}}$ for a basic system, it implies that its corresponding $S N R_{\text {eirp }}$ value is upper bounded by $\frac{n_{T} P_{T x}}{\sigma_{n}^{2}}$.

\section{Simulation Results and Analysis}

\subsection{Simulation Process and Parameters}

The simulated system is composed of one access point equipped with multiple antennas (linear array of $0 \mathrm{dBi}$ omnidirectionnal and vertically polarized antennas), and two receivers. Each receiver has two $0 \mathrm{dBi}$ omnidirectionnal antennas. By default, the antenna spacing is set to $0.5 \lambda$. A Matlab source code [11] was used to compute the different 802.11ac TGac-B channel samples over a $20 \mathrm{MHz}$ bandwidth. To have 
representative results, 100 couples of users $(K=2)$ are randomly drawn around the access point following the IEEE TGac recommendations [9]. For each drawing, we use a simulation length equal to 55 coherence times of the MIMO channel to simulate the fading. By setting the "Fading Number of Iterations" in the Matlab channel model to 512, 488 interpolated channel samples are collected for each couple of users to simulate 10 fading periods.

All the results are compared to an i.i.d Rayleigh channel and presented in terms of the MU-MIMO to SU-MIMO capacity ratio.

\subsection{Results for Equal Power Allocation}

Figures 2 and 3 display the simulation results in terms of the MU-MIMO to SUMIMO capacity ratio for basic and eirp-equal systems. Average values, $10 \%$ and $90 \%$ quantiles $\left(\mathrm{q}_{10}\right.$ and $\left.\mathrm{q}_{90}\right)$ are represented to estimate the confidence intervals. $S N R_{\text {eirp }}$ is set to $20 \mathrm{~dB}$ for eirp-equal and $S N R$ for basic is also set to $20 \mathrm{~dB}$. $n_{T}$ varies from 4 to $10 \mathrm{Tx}$ antennas.

These figures show that the MU to SU-MIMO capacity ratio increases with the number of transmit antennas for TGac-B and Rayleigh channels. The ratio changes from 1.2 to 1.77 for the eirp-equal system in a residential environment, which represents more than $50 \%$ of capacity gain. Note that the gain without the EIRP constraint is around $45 \%$. It has been shown in previous studies that increasing the number of transmit antennas favorably impacts the capacity gain on an i.i.d Rayleigh channel under SNR constraint [4], [6]. We have been able to prove that this result holds even under the EIRP constraint and with correlated channels as in TGac models. The difference between the the $10 \%$ and $90 \%$ quantiles reduces as the number of antennas increases. This shows that fading has less impact on the capacity values which become less scattered.

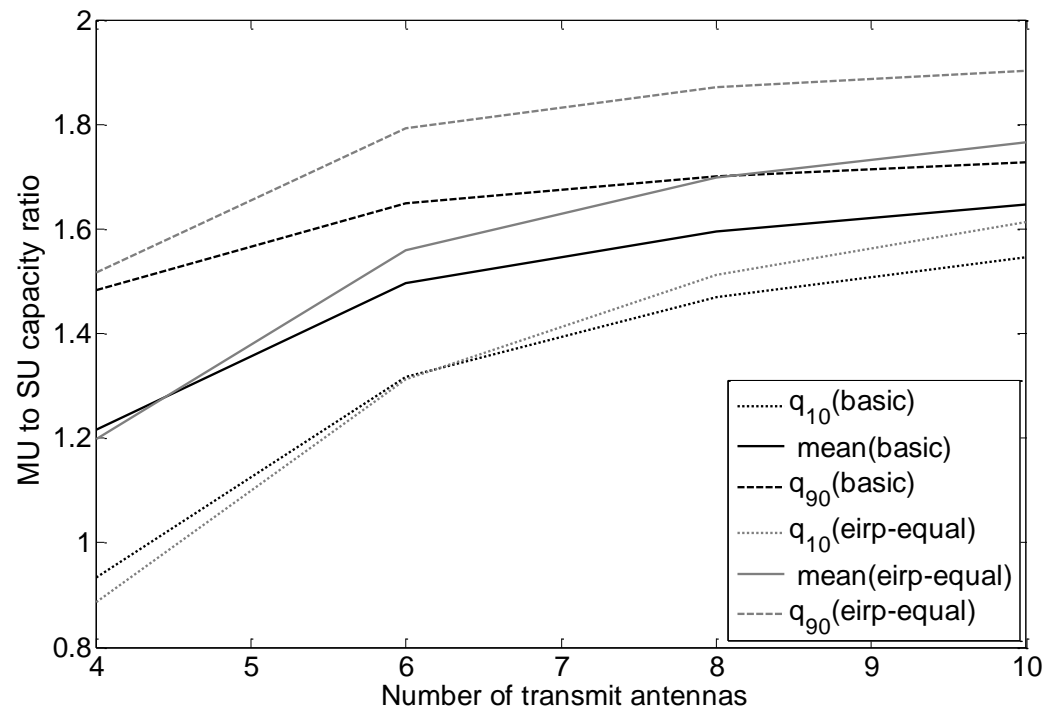

Fig. 2. MU to SU capacity ratio for an IEEE TGac-B channel (residential). 


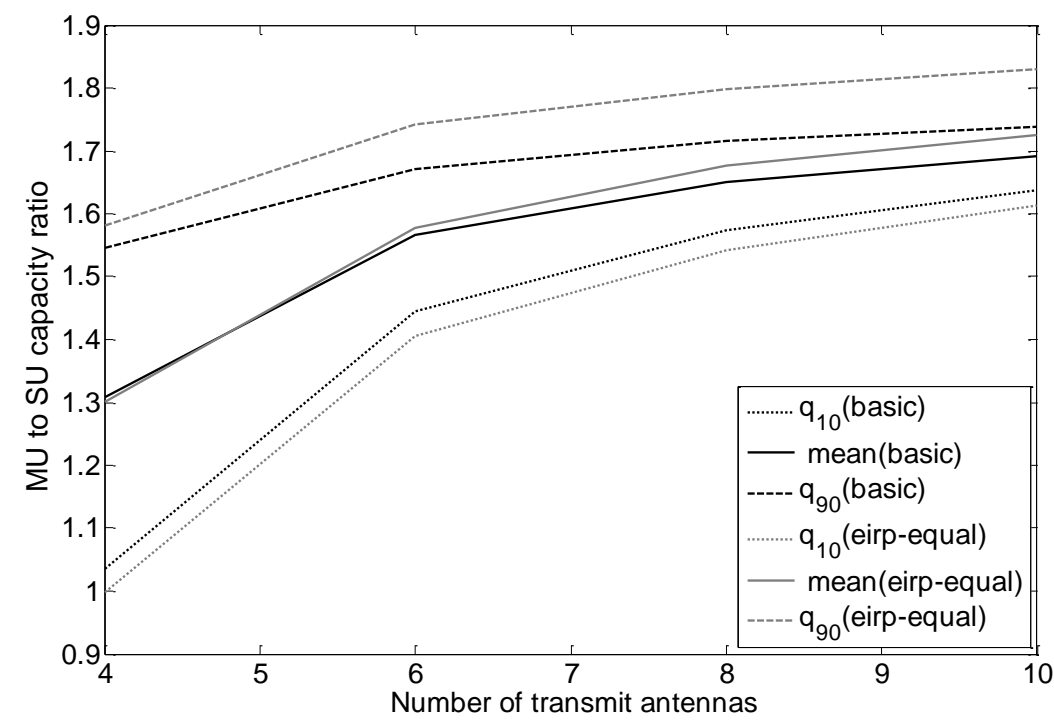

Fig. 3. MU to SU capacity ratio for an i.i.d Rayleigh channel.

The basic and eirp-equal comparisons are biased. In fact, a system relying on a total Tx power does not satisfy a constant EIRP constraint since it may have an increasing EIRP as $n_{T}$ increases. Intuitively, we could expect that for a basic system, the MU-MIMO to SU-MIMO capacity ratio increases more rapidly in function of $\mathrm{n}_{\mathrm{T}}$ than for an eirp-equal system, but simulations prove the opposite. In fact SUMIMO takes advantage of the power $P_{e}$ when the system is not under EIRP constraint. For instance in our simulated case $(K=2)$ where $N S S=4$ for MU-MIMO and $N S S=2$ for each one of the single users, the EIRP reached by the MU-MIMO system $\left(E I R P_{M U}\right)$ is expressed as $E I R P_{M U}=\frac{P_{T x}}{N S S}\left[\max _{\theta}\left(a(\theta)^{*} \bar{W} W^{t} a(\theta)\right)\right]$ and is upper bounded by $n_{T} \frac{P_{T x}}{4}$. Similarly, for the same value of $P_{T x}$, the single user EIRP is upper bounded by $n_{T} \frac{P_{T x}}{2}$. This means that for a system under the same EIRP constraint, the allocated power tends to be lower for SU-MIMO than for MU-MIMO. For the basic system, the allocated power is the same for SU-MIMO and MU-MIMO. Fig.4 shows the average capacity value for MU and SU. It is well observed that the SU capacity increases rapidly with $n_{T}$.

\subsection{Impact of Power Allocation Strategy}

To check whether the MU-MIMO capacity always outperforms the SU-MIMO, we have evaluated the probability when the MU-MIMO capacity is lower than the SUMIMO capacity. This is illustrated in Fig.5 for eirp-equal and eirp-unequal schemes versus the number of transmit antennas.

Fig. 5 shows that there are some channel snapshots for which the MU-MIMO is less efficient than SU-MIMO in terms of capacity, particularly when $n_{T}$ is equal to 4 . The probability reaches $20 \%$ for equal power repartition between spatial streams. 
However, the unequal power repartition decreases this probability by $6 \%$. We also observe that the MU-MIMO capacity gain for unequal repartition is slightly greater than the one observed for a fair power distribution. Nevertheless, the gain is not significant: we have around $3 \%$ of capacity gain by contrast to high computational complexity. The probability is almost $0(\leq 1 \%)$ for $n_{T}=6$ and is equal to 0 for $n_{T} \geq 8$. These results can be explained by examining the overall system: for $n_{T}=4$, the MU-MIMO system is composed of 4 antennas in the transmit and the receive sides with 4 spatial streams. This gives no diversity possibilities. The number of spatial streams becomes strictly less than the number of Tx antennas since $n_{T}$ increases. As a result, the system takes benefit from the transmit diversity and thus shows probabilities which tend to 0 .

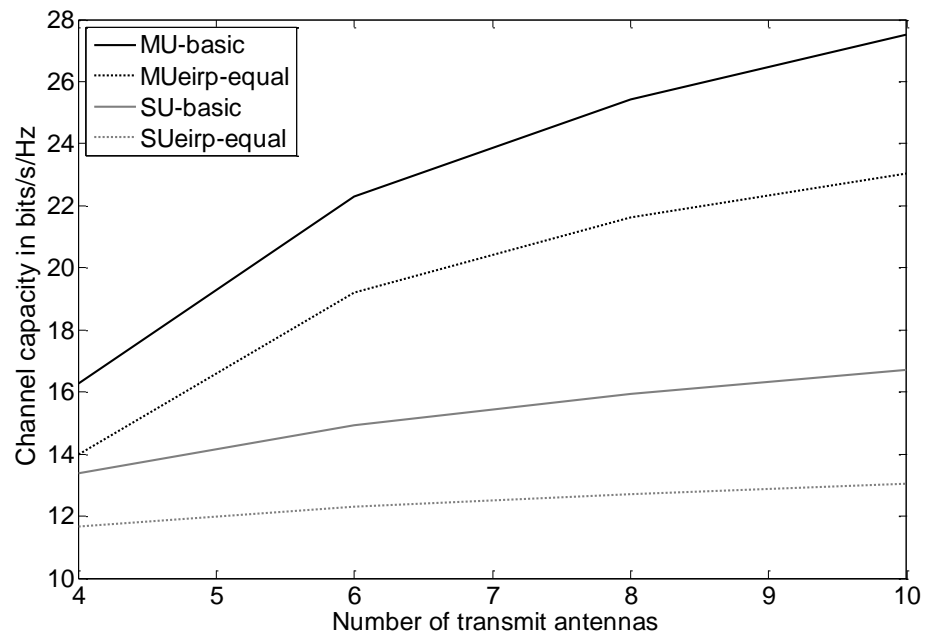

Fig. 4. Capacity value achieved by the basic and eirp-equal systems

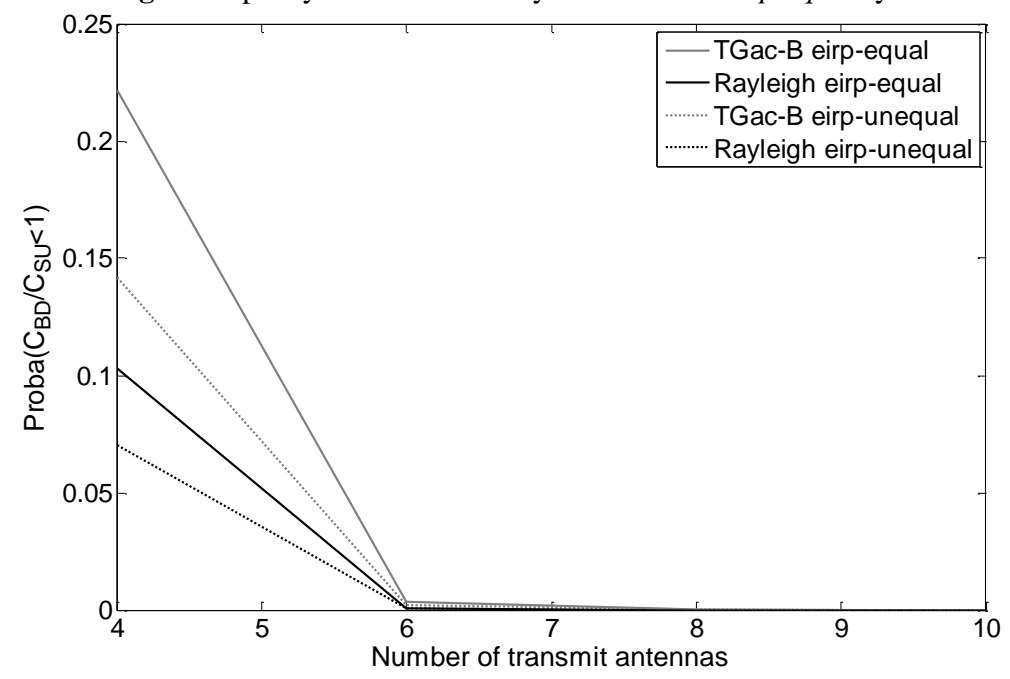

Fig. 5. Proba $\left(\mathrm{C}_{\mathrm{BD}} / \mathrm{C}_{\mathrm{SU}} \leq 1\right)$ versus the number of transmit antennas 


\section{Conclusion}

In this paper, we have formulated an optimization problem for 802.11ac MU-MIMO systems, considering multiple spatial streams for each user and taking into account the EIRP constraint. Then we have derived numerical results for a typical home network environment and correlated 802.11ac channel models. Two transmit power allocation methods have been evaluated: equal and unequal repartition based under the same EIRP constraint. We have compared these two strategies with the more common MUMIMO under the total Tx power constraint. We have shown that MU-MIMO precoding takes benefit from the EIRP constraint. In particular, we have shown that under EIRP constraint, it is recommended to have a transmit antenna number greater than the total number of spatial streams to guarantee a MU-MIMO capacity gain over SU-MIMO.

In further work, MU-MIMO measurements will be performed to validate these results with real propagation channels.

\section{References}

1. 802.11ac-2013 - IEEE Standard for Information technology-- Telecommunications and information exchange between systems - Local and metropolitan area networks -- Specific requirements -- Part 11: Wireless LAN Medium Access Control (MAC) and Physical Layer (PHY) Specifications -- Amendment 4: Enhancements for Very High Throughput, (2013).

2. Saavedra, J.: Multidiffusion et diffusion dans les systèmes OFDM sans fil. Thèse de doctorat d'état, Univ. Paris-Sud, Paris, France, (2012).

3. Zhao, L., Wang, Y., Charge, P.: Efficient power allocation strategy in multiuser MIMO broadcast channels. Personal Indoor and Mobile Radio Communications (PIMRC), pp.2591-2595, (2013).

4. Spencer, Q.H., Swindlehurst, A.L., Haardt, M.: Zero-forcing methods for downlink spatial multiplexing in multiuser MIMO channels. IEEE Trans. on Signal Proc., vol. 52, no. 2, pp. 461-471, (Feb. 2004).

5. Kuzminskiy, A. M.: Downlink beamforming under EIRP constraint in WLAN OFDM systems. Signal Processing, vol. 87(5), pp. 991-1002, (May 2007).

6. Choi, L-U, Murch, R.D.: A transmit preprocessing technique for multiuser MIMO systems using a decomposition approach. IEEE Trans. on Wireless Comm., vol. 3, no. 1, pp. 20-24, (Jan. 2004).

7. Breit, G., Sampath, H., Vermani S. et al.: TGac Channel Model Addendum Support Material. Mentor IEEE, Doc IEEE 802.11-09/06/0569r0, (May 2009).

8. Erceg, V., Schumacker, L., P. Kyritsi P. et al.: TGn Channel Models. Mentor IEEE, Doc IEEE 802 11-03/940r4, (May 2004).

9. Bouhlel,A., Guillet, V., El Zein, G., Zaharia, G.: Transmit Beamforming Analysis for MIMO Systems in Indoor Residential Environment Based on 3D Ray Tracing. Springer Wireless Personal Communications, (Dec 2014), pp.1-23.

10. Grant M. and Boyd S.: Matlab Software for Disciplined Convex Programming http://cvxr.com/cvx/, (March 2014).

11. Schumacher L., Dijkstra, B.: Description of a Matlab implementation of the indoor MIMO WLAN channel model proposed by the IEEE 802.11 TGn Channel Model SpecialCommittee. Implementation note, version 3.2, (May 2004). 